\title{
The Trajectory Synthesizer Generalized Profile Interface
}

\author{
Alan G. Lee * \\ NASA Ames Research Center MS 210-8, Moffett Field, CA, 94035 \\ Xavier Bouyssounouse ${ }^{\dagger}$ \\ NASA Ames Research Center MS 269-3, Moffett Field, CA, 94035 \\ and \\ James R. Murphy * \\ NASA Ames Research Center MS 210-8, Moffett Field, CA, 94035
}

\begin{abstract}
The Trajectory Synthesizer is a software program that generates aircraft predictions for Air Traffic Management decision support tools. The Trajectory Synthesizer being used by researchers at NASA Ames Research Center was restricted in the number of trajectory types that could be generated. This limitation was not sufficient to support the rapidly changing Air Traffic Management research requirements. The Generalized Profile Interface was developed to address this issue. It provides a flexible approach to describe the constraints applied to trajectory generation and may provide a method for interoperability between trajectory generators. It also supports the request and generation of new types of trajectory profiles not possible with the previous interface to the Trajectory Synthesizer. Other enhancements allow the Trajectory Synthesizer to meet the current and future needs of Air Traffic Management research.
\end{abstract}

\section{Introduction}

$\mathrm{T}$ HE Next Generation Air Transportation System (NextGen) project was created by the Joint Planning and Development Office (JPDO) to develop future Air Traffic Management (ATM) concepts that will accommodate the expected future increase in traffic levels and improve the efficiency of the National Airspace System (NAS). ${ }^{1}$ As a core member of the JPDO, the National Aeronautics and Space Administration (NASA) initiated the Airspace Systems Program to research and develop NextGen concepts. Interoperability Research, a research focus area under this program, includes the study of methods for computing accurate aircraft trajectory predictions that are interoperable with trajectories generated by aircraft flight management systems (FMS). ${ }^{2}$ Trajectory prediction requires an understanding of the aircraft performance and the constraints (e.g. altitude and speed restrictions) that govern the trajectory. NASA proposed the development of a trajectory predictor with the following capabilities ${ }^{3}$ :

- Support variable-order trajectory constraints

- Describe trajectory constraints which can support interoperability with other trajectory predictors (and FMSs)

- Support air and ground predictions of departure, en-route, and arrival transition segments

Accurate trajectory predictions are necessary for ATM functions such as conflict prediction, conflict resolution, and flight scheduling. Aircraft trajectory predictions are based on information from aircraft performance data, sitespecific airspace information (e.g., waypoints), weather information, and initial aircraft state conditions and constraints (e.g. altitude and speed restrictions). These constraints are used in concert with aircraft equations of motion to predict the aircraft's trajectory. Many trajectory predictors restrict the number or location of constraints due to the complexity involved with creating a wide variety of trajectory types. ${ }^{4}$ For example, NASA's Center-

\footnotetext{
* Aerospace Engineer, Aerospace Simulation Research \& Development, MS-210-8, non-member.

${ }^{\dagger}$ Software Engineer, Intelligent System Division, M/S-269-3, non-member.

* Software Manager, Aerospace Simulation Research \& Development, MS-210-8, Senior Member.
}

1

American Institute of Aeronautics and Astronautics 
TRACON Automation System (CTAS) Trajectory Synthesizer (TS) ${ }^{5}$, which was used to provide predictions for the Traffic Management Advisor (TMA) ${ }^{6,7}$ modeled only six distinct types of en-route climb and descent profiles. A more generalized application of air traffic constraints is needed to support the ATM research involving more complex automated and controller assisted aircraft maneuvers ${ }^{8,9}$ as well as future trajectory predictor interoperability.

Trajectory predictor interoperability with an aircraft FMS could allow detailed trajectory information to be conveyed between an ATM Decision Support Tool (like TMA) and the aircraft. Aircraft intent information could be sent from the aircraft to the Decision Support Tool (DST) allowing the DST to obtain more accurate trajectory predictions. Conversely, the DST could send the aircraft advisories with details to perform complex aircraft maneuvers.

Interoperability between various trajectory predictors would enable the same set of trajectory constraints to be processed by different predictors. Various computation models are used for trajectory predictors that result with differences in trajectory accuracy and computation speed. A DST may have specific accuracy and computation speed requirements. Interoperability would enable a DST to easily replace the trajectory predictor it uses with one that met these requirements.

The Generalized Trajectory Profile (GenProf) framework was developed to directly address the expression of variable-order constraints and trajectory interoperability requirements ${ }^{2}$. The GenProf framework offers a systematic methodology for specifying and calculating trajectories with multiple spatially-separated interdependent constraints. GenProf separates the equations of motion from the constraint dependencies by providing a scripting language that specifies the trajectory constraints independent of the method for calculating the path between the constraints. The GenProf framework was successfully applied to describe the vertical and speed constraints for the CTAS TS, supporting the more demanding research requirements of the NextGen Airspace Program., ${ }^{9,10,11}$

This paper describes the development and testing of the new GenProf interface and associated modifications. The limitations of the previous Baseline TS that served as the motivation for this new interface, and the capabilities of the new GenProf interface are discussed. Finally, the resultant trajectories that can be modeled by the Baseline and GenProf TS are compared.

Since the purpose of the GenProf development was to expand TS capability, the focus of this work was on comparing trajectory predictions generated by the Baseline and GenProf TS. The accuracy of the trajectory predictions was not compared with actual aircraft track data. The GenProf interface is also applicable to trajectories generated for aircraft in the terminal airspace, however, to limit the scope of the paper, the discussion focuses on trajectories generated for aircraft in the en-route airspace.

\section{Background}

Trajectory generation capability, provided by the TS, is critical to ATM research at NASA Ames. The TS computes 4-D (three physical dimensions and time) aircraft trajectories using equations of motion based on a simplified point mass aircraft model ${ }^{4}$. While the basic trajectory generation algorithm has not changed significantly over the years, the overall TS software has gone through several enhancements necessary to meet NextGen research requirements.

\section{A. Trajectory Synthesizer Development History}

The first incarnation of the TS was referred to as the Descent Advisor (DA). Written in FORTRAN77 and completed in 1984, the DA predictions were used to study the efficacy of controller advisories for fuel-efficient descents to the meter fix. ${ }^{12,13}$ From a design standpoint, the software was completely integrated; DA handled the pilot intent modeling as well as the trajectory generation. Pilot intent modeling describes how the aircraft is operated, such as climbing to a specific altitude. The trajectory generator calculates the aircraft's trajectory based upon pilot intent information and models of atmospheric conditions, aircraft performance, and aircraft flight dynamics.

By 1993, DA was integrated with the CTAS program, renamed the Trajectory Synthesizer (hence TS), and rewritten in the $\mathrm{C}$ programming language. ${ }^{14}$ At this time, the software was redesigned to split the trajectory engine from the routing and intent decision logic. This version of TS was used by NASA and the FAA to develop the TMA system, which coupled the predictions at the meter fix (and runway) with a scheduling algorithm to provide controllers with delay advisories for arrivals. ${ }^{15}$ This version of the TS also supported research by Green and Vivona ${ }^{16}$ that measured the prediction accuracy of the descent trajectories to the meter fix for a wide variety of test conditions. 
As trajectory generation requirements continued to evolve based on the advanced descent and conflict prediction algorithms being researched, ${ }^{17,18}$ the TS was rewritten again, this time in $\mathrm{C}++$. This effort resulted in the Baseline TS with many of the benefits inherent with Object Oriented design, such as code reuse, code extensibility, and improved maintainability. However, it retained much of the older logic that determined the constraints on the aircraft trajectories. The Baseline TS uses a set of pre-defined vertical flight profile shapes containing a set of rigid constraint combinations. It is, therefore, difficult to add new profiles using the Baseline TS architecture. Given the wide range of possible profiles that an aircraft can fly, this is a significant disadvantage to the ATM research community. The Baseline TS software is being used by the FAA operational TMA system under Free Flight Phase $1 .{ }^{19}$

Although the TS software has gone through many design iterations, its core trajectory algorithms have remained intact, providing a consistent platform to conduct research and support operational systems. The trajectory engine has been repeatedly validated through research, and operationally via the deployed TMA system ${ }^{16,17,20}$. The GenProf interface is the next phase of redesign of this software. It builds upon the existing trajectory engine to better support anticipated NextGen research requirements.

\section{B. Design Philosophy of the Trajectory Synthesizer}

Many trajectory generators like the TS use a layered software architecture (Figure 1). In the TS, the top layer is the client application such as a DST that formulates the constraints and initial conditions and then sends the trajectory request to the trajectory generator (Figure 1). The TS trajectory generator itself has two layers. The first one is the behavioral model, which models the pilot or controller intent. The second layer is the math model, which consists of the aircraft equations of motion, aircraft performance model data, and control laws. Figure 1 shows a diagram illustrating this concept.

Each of the TS redesign efforts, discussed previously, reevaluated the functional responsibilities of the TS and those of the client application. This was trivial with the $1984 \mathrm{DA}$ software where the trajectory engine and client functionality were integrated. With each successive TS software iteration, the ideal division of responsibilities between the client application and TS became more clearly defined. In their research, Vivona et. al. outline a set of rules for any trajectory prediction software to help make these determinations. ${ }^{21}$

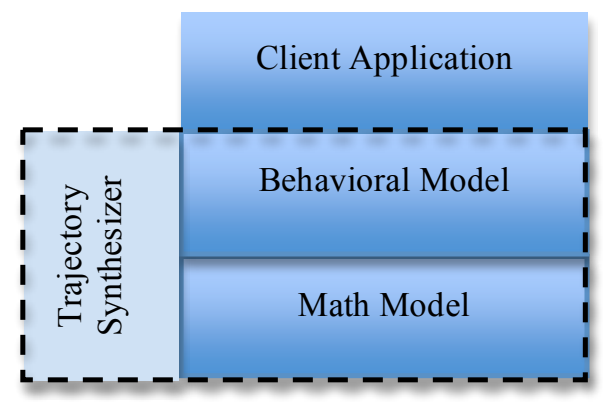

Figure 1: General Layered Structure of the TS

The philosophy behind the current TS enhancement was to make the software architecture flexible. The TS is a single codebase that must be agile enough to keep up with the changing research requirements and serve the specific requirements of the many client applications such as controller DST concepts being developed. To help ensure that different DST functionality does not conflict with each other, the Trajectory Predictor (TP) boundary rule is used. The TP Boundary Rule is "...if the capability directly supports a key function of the client application, then the capability is considered a client application capability and outside the scope of the TP. $" 21$

Applying the TP Boundary Rule to the design of the TS allows the software to have flexibility it needs to serve multiple DSTs. In particular, moving the decision-making process involved in determining the constraints of the desired trajectory to the client application, allows the TS to function analogously to a calculator. The TS simply receives inputs and provides an output trajectory solution to be used as the client sees fit, without regard to the specific motivation behind it.

This design philosophy also provides a level of transparency for creating the trajectory solution. For example, the Baseline TS software contained logic to modify the descent speed and flight path angle of the aircraft to ensure that adapted altitude constraints are satisfied. With the new modifications, the TS would make adjustments to the TS request without any notification to the client. When implemented, these adjustments supported enhancements to the TMA program to support the situation where the altitude and speed of the aircraft at the meter fix is known so a 
descent to those constraints without regard to other constraints is acceptable. However, the lack of notification regarding adjustments to the TS request reduced the usability of the resultant trajectory solutions because other clients did not know all of the assumptions used to generate the trajectory. For example, DSTs employing conflict detection and conflict resolution are extremely concerned about the aircraft position and time throughout the trajectory. Hence, the path of the aircraft is just as important for these clients as the time it reaches a particular fix. This highlights the different requirements that DSTs need from the trajectory generator and the need to use the TP Boundary Rule in the TS enhancement.

As mentioned earlier, the Baseline TS adjusted various inputs of the trajectory request such as speeds or flight path angles as necessary to compute the trajectory. This is necessary because not every trajectory request will initially result with a successful trajectory calculation. Trajectory calculations may fail when the aircraft model performance capabilities are exceeded. In the enhanced TS, the code for handling these trajectory failures has been moved out of the TS and made into a more flexible organized mechanism. This allows more transparency and control by the client application as to how the failure should be addressed. When the client application for the TS involves fundamental research it may be better to have the TS fail to help understand why the trajectory request constraints could not be satisfied. When the TS is used to support a DST for prototyping and simulation purposes, relaxing and adjusting constraints maybe allowed since there is greater emphasis on computing a successful trajectory at the cost of loss of accuracy on the constraints.

The ability to allow expression of trajectory constraints in a flexible manner was a major design goal of the TS enhancement. Actual arrival profiles may involve a number of level-offs (down to the meter fix and throughout the terminal airspace) or, with the availability of such concepts as Continuous Descent Arrivals, none at all. ${ }^{22}$ Figure 2 shows a descent trajectory with multiple level-offs. Since the TS is primarily used to test these and other ATM concepts, it is critical that it be able to model a wide variety of constraints. Hence, the primary design principle of the GenProf interface was the decoupling of the vertical constraints from the trajectory engine by providing an interface for entering and interpreting the constraints in a form that can be used as input to the trajectory predictor. To express the constraints in a flexible manner a scripting language was devised that was intuitive to use.

Besides flexibility, the GenProf scripting language was designed to be generic. By being generic the language is not directly tied to a specific TP and could potentially be used by others. This allows the GenProf language to be a medium for interoperability used in expressing vertical constraints between different TPs and clients. Every TP has different characteristics that it models and also limitations to its performance and accuracy. Interoperability provides the flexibility for client applications using a common interface to swap TPs to meet client requirements.

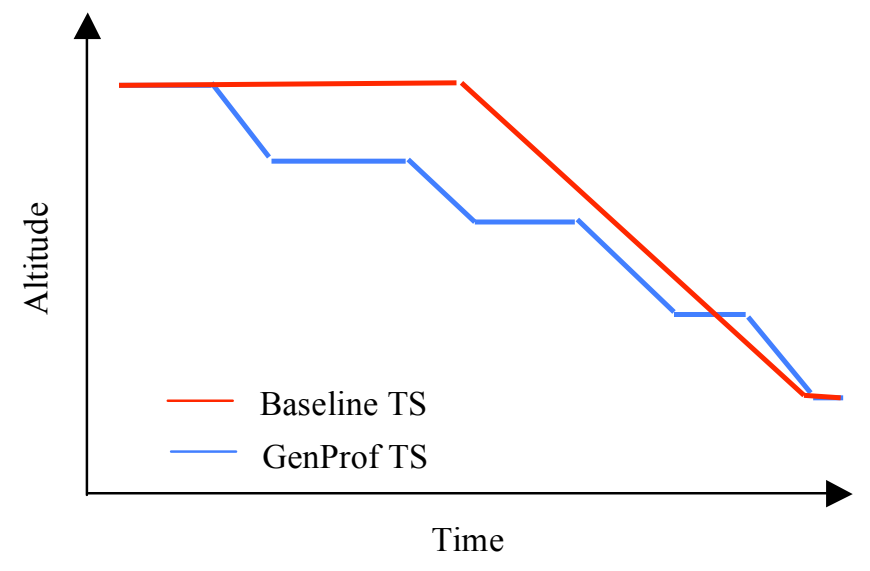

Figure 2: An example of a multiple step down trajectory that can be modeled using GenProf versus a single step trajectory model limitation by the older version of TS interface

\section{Generalized Profile Interface}

An aircraft trajectory is generated using its initial condition and trajectory constraints. The most basic elements of the aircraft's initial condition are weight, latitude-longitude position, altitude, heading, and speed (ground speed, calibrated airspeed, true airspeed, or Mach number). Radar track data is used to supply the TS with the aircraft's 
initial latitude-longitude position, altitude, heading, and ground speed. In the case of the TS, the aircraft weight is obtained from a database of aircraft models. Airspeeds are determined by using the ground speed and weather data.

The constraints are the various conditions that must be attained for the trajectory to be successful. For a trajectory prediction, these constraints are usually determined using the aircraft's flight plan. For a trajectory advisory, the constraints are determined from a conflict resolution related to a conflict prediction. Constraints are categorized as being related to either the vertical or horizontal portion of the trajectory. The constraints for the horizontal portion of the trajectory are the list of waypoints that the aircraft uses to define its horizontal flight path, i.e. the aircraft's ground track. From this flight path, a single path distance can be determined. Points along this path are used to bind the latitude-longitude position with the vertical position of the trajectory. The software to generate the horizontal portion of the flight path is common to both the Baseline TS and the new GenProf TS. Details of the horizontal flight path calculation are provided by Erzberger and Lee ${ }^{4}$ as well as Slattery and Zhao 5 .

The GenProf Trajectory Interface allows the user to express a set of vertical constraints that is used by TS integrator function. It uses a scripting language to describe the vertical constraint inputs. This language provides the flexibility to compose any number of constraint combinations. There are three basic GenProf elements: the Anchor, the Profile, and the Stop. GenProf incorporates specifies rules of syntax for combining these elements. The following section discusses the GenProf scripting language elements and syntax.

\section{A. GenProf Elements}

The GenProf Anchor holds the state information that typically represents the initial conditions of the aircraft trajectory. The GenProf Anchor consists of an altitude, a speed, and a horizontal path distance. The speed can be specified as a Mach, CAS, true airspeed, or ground speed. The TS converts among these speeds by interpolating atmospheric and wind data from the NOAA RUC model. ${ }^{23}$ The horizontal path distance is the length of the ground path of the resultant trajectory. It is derived by integrating the straight line and curved segments that comprise the route of flight.

The GenProf Stop element represents the capture condition that terminates the trajectory segment. GenProf can be used to specify an arbitrarily complex boolean expression of stopping conditions with any number of AND $(\cap)$ and OR $(\cup)$ expressions. However, the current Vertical Profile implementation of GenProf limits the expressions which can be evaluated to using only OR expressions, each of which captures a specific value for one of the following variables:

- Altitude Capture

- Time Capture

- Path Distance Capture

- Speed Capture (Mach, CAS, TAS, or ground speed).

The GenProf Profile element is the most complex of the three. It describes how a trajectory segment is built from either a GenProf Anchor or Stop to the next Stop element by combining a set of parameters for the aircraft to adhere to until a given capture condition (or set of capture conditions described by the GenProf Stop) is met. Currently these parameters include the flight path angle, vertical speed, engine throttle setting, and Mach-CAS profile. Setting two of these parameters dictates the equations of motion that will be used to compute the trajectory starting from the Anchor until the Stop is reached.

The Mach-CAS profile is the speed schedule that is typically followed by jets when the aircraft climbs or descends. During a descent (starting from an altitude where the pilot is initially flying using Mach speed) a pilot will initially maintain the Mach speed described by the Mach-CAS profile. While maintaining the Mach speed, the CAS increases as the altitude decreases. When the CAS value designated by the Mach-CAS profile is reached, the pilot will switch from maintaining the Mach profile speed to the CAS profile speed. A climb would use the reverse of this procedure. Obviously the Mach-CAS profile is applicable to a segment with an altitude change and does not apply to a cruise trajectory segment. Currently the Mach-CAS profile must be set as one of the two parameters for the Profile. In the future the GenProf Profile will remove this requirement and allow any combination of two parameters to be specified.

\section{B. GenProf Syntax}

The simplest GenProf expression describes a single trajectory segment. This consists of three elements, an Anchor, followed by a Profile, which in turn is followed by a Stop. For example, for an aircraft flying at a cruising altitude of 33,000 ft and holding a speed of Mach 0.8 for 20 minutes, the GenProf expression is: 


\begin{tabular}{|c|c|c|}
\hline Anchor & Profile & Stop \\
\hline $\begin{array}{c}\text { 33K ft Altitude } \\
\text { Mach 0.8 }\end{array}$ & Mach 0.8 & $20 \mathrm{~min}$ \\
\hline
\end{tabular}

Table 1: A Simple GenProf Expression

The scripting language allows for more Profile-Stop pairs to be chained together, extending the vertical profile as necessary and allowing complicated vertical profile expressions to be created. For example, if the previous example is expanded to include an additional GenProf Profile having a Fixed Idle Engine Control descent to 18,000 ft using a Mach-CAS Profile of Mach 0.8 / 280 knot CAS, the following GenProf expression is used (with the added profile described as Profile \#2 and Stop \#2 respectively):

\begin{tabular}{|c|c|c|c|c|}
\hline Anchor & Profile \#1 & Stop \#1 & Profile \#2 & Stop \#2 \\
\hline $\begin{array}{c}\text { 33K ft Altitude } \\
\text { Mach 0.8 }\end{array}$ & Mach 0.8; & $20 \mathrm{~min}$ & $\begin{array}{c}\text { Mach 0.8 / 280 knot CAS; } \\
\text { (ixed Idle Engine Control }\end{array}$ & 18K ft Altitude \\
\hline
\end{tabular}

Table 2: A Simple GenProf Expression Appended with an Additional Descent Segment

This GenProf expression results in a Cruise-Decent vertical profile. When the first segment of this GenProf expression (the Anchor, Profile \#1, Stop \#1) is solved, the capture conditions of Stop \#1 are achieved, and the remaining aircraft state variables are calculated. At this point Stop \#1 has the complete aircraft state information and has become equivalent to an Anchor. This new Anchor is used to solve the next trajectory segment described by Profile\#2 and Stop \#2. This procedure is repeated for any additional Profile-Stop pairs. Figure 3 is a notional diagram showing the GenProf Expression used in Table 2.

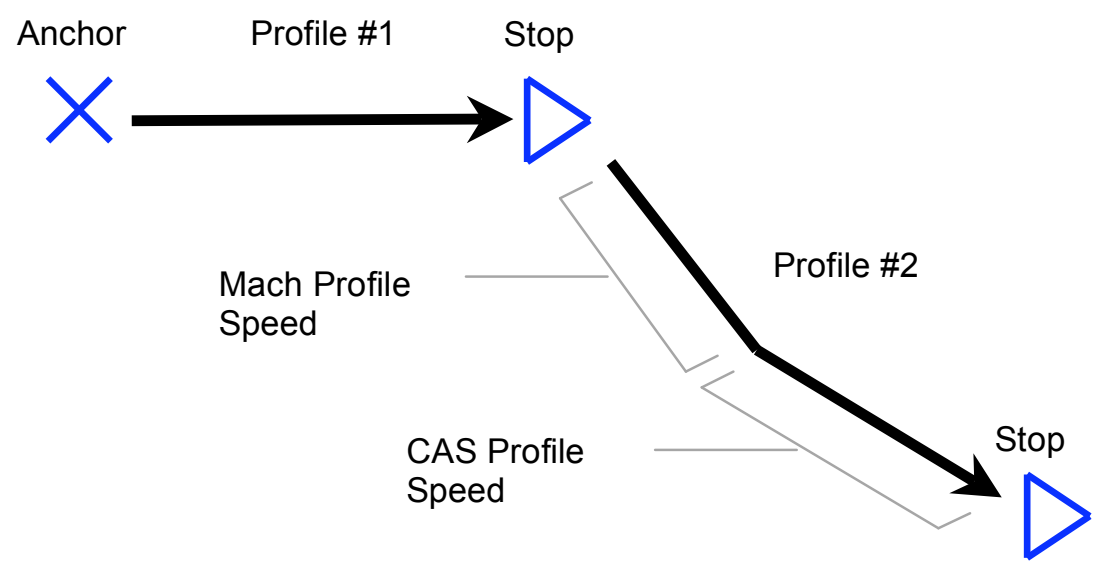

Figure 3: Graphical Representation of the GenProf Expression used in Table 2

\section{Generalized Profile Interface Implementation}

For the most part, the underlying trajectory integration functionality is the same for GenProf as it is for the previous Baseline TS. GenProf can describe the same trajectory constraints as the previous version of the TS but has capability to describe more. The GenProf version of the TS has evolved into an architecture containing more smaller separate software modules than the Baseline TS. This allows for easier replacement and modification of functionality and, therefore, has the flexibility needed to accommodate the requirements of the different DSTs.

The GenProf implementation is still under development. GenProf uses the Extensible Markup Language (XML) data format to allow data elements to be easily added to the GenProf scripting language. The following section will describe the TS Input in more detail and then discuss the TS processing flow. 


\section{A. TS Input}

The TS Input data structure is the vehicle used to relay the client application's trajectory request to the TS. The XML data format for the input was chosen to provide for potential interoperability with other Trajectory Predictors (TPs) other than the TS. The XML data format is a self-describing data format allowing it to be a suitable format for data interchange. Another benefit to the XML data format is that it is also easily recognizable text allowing users to quickly examine or edit the input. One disadvantage, however, is that the data format can be very verbose and, therefore, occupy a large amount of disk space. A sample of the GenProf XML input for the expression in Table 1 is shown in Figure 4.

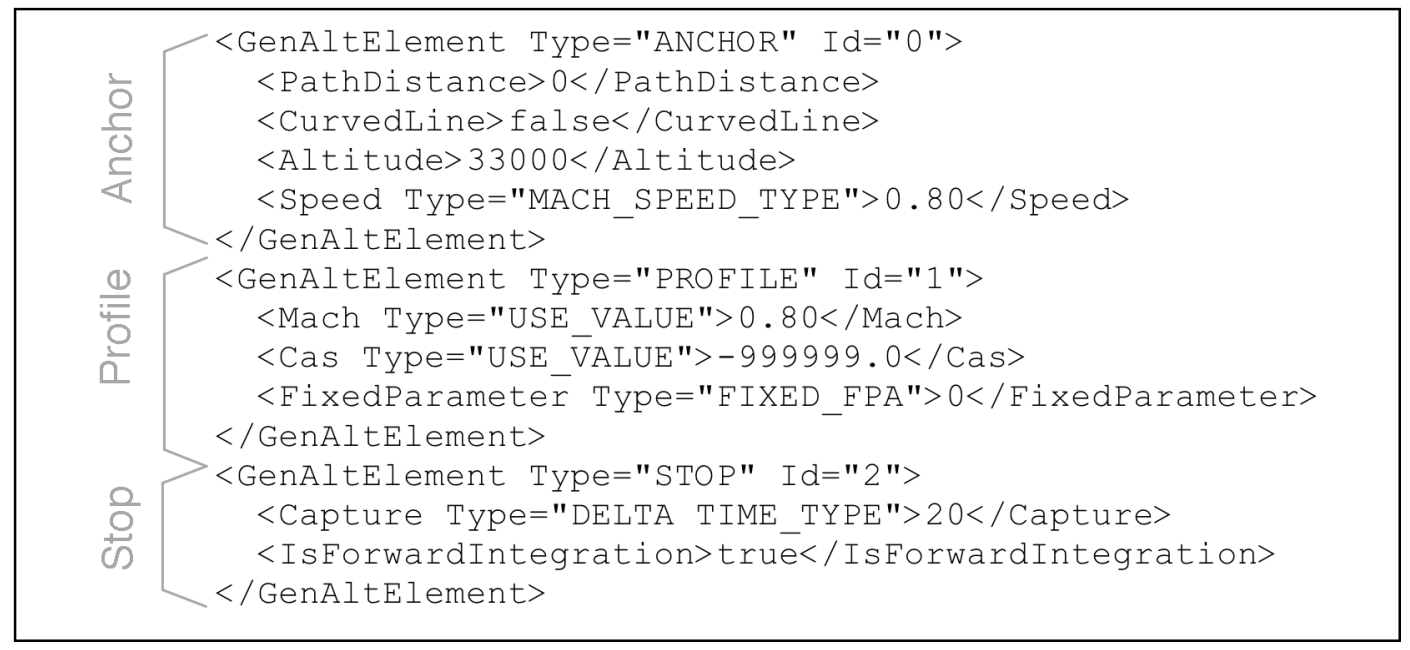

Figure 4: GenProf XML Input for a Cruise Trajectory

\section{B. TS Input Processing}

The current implementation of the processing flow for the TS takes the client data inputs to the Baseline TS and then translates them into a set of GenProf inputs. A TS Input request will contain both a Baseline set of inputs and a GenProf set. This was done in order to compare the results generated by the two different methods. The Baseline to GenProf translation process will eventually be removed allowing a direct flow from the client to the GenProf interface.

After the translation process, the constraints are analyzed and the Horizontal profile is processed. Then depending on the Vertical Profile processing mode, GenProf or Baseline Vertical Profiles are processed. The final step is the trajectory integration process. A diagram of this process flow is shown in Figure 5.

\section{Comparisons with the Baseline TS}

The Baseline TS generates six well defined vertical profiles: Ascent Cruise, Descent Cruise, Ascent Cruise Descent, Transition Altitude Descent, Transition Altitude Ascent, and Level Flight (See Figure 6). These were the only vertical profiles that could be created. This limitation was one of the primary forces driving the design and development of the GenProf interface. Additional features of the GenProf elements were added to allow flexibility in describing the climb and descent transitions. The Baseline TS and the GenProf TS were compared to ensure that GenProf is able to continue to produce the existing set of trajectories, while allowing for new types of trajectory profiles. For any given trajectory request, however, a successful trajectory from both the Baseline TS and GenProf TS does not necessarily mean that the two trajectories are identical. The remainder of this section describes the comparisons and explains these differences. 


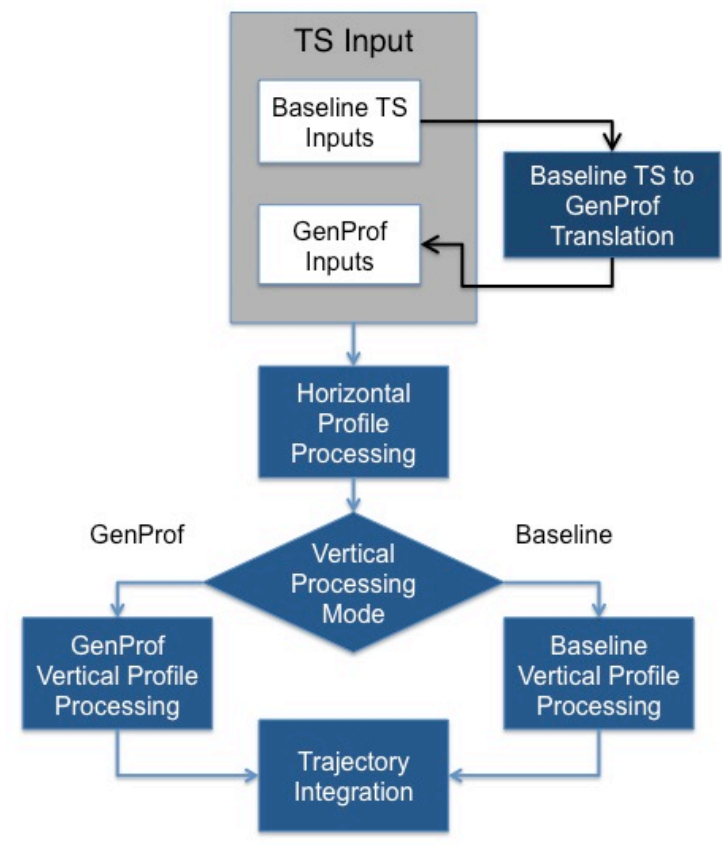

Figure 5: TS Input Processing Flow

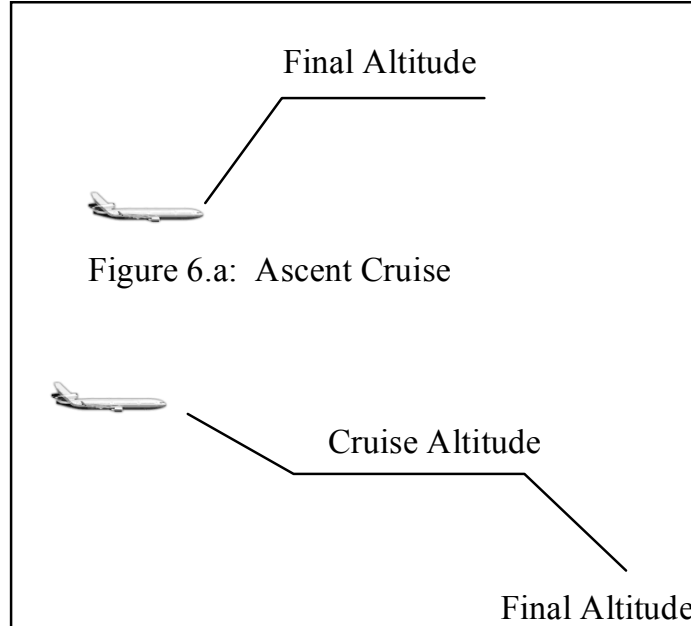

Figure 6.c: Transition Altitude Descent

Final Altitude

Figure 6.e: Level Flight

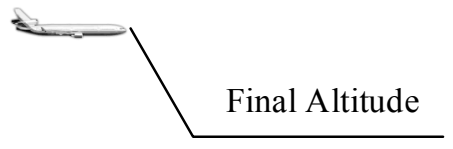

Figure 6.b: Descent Cruise

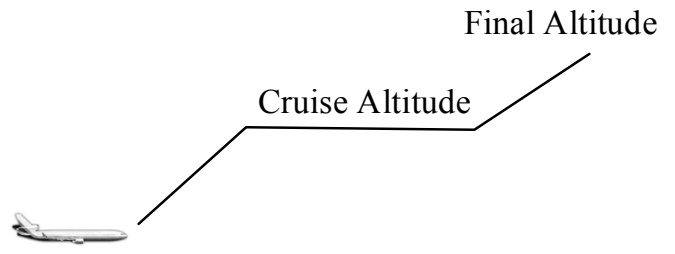

Figure 6.d: Transition Altitude Ascent

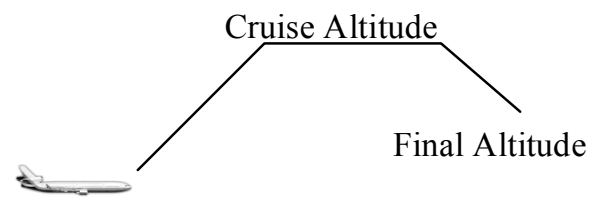

Figure 6.f: Ascent Cruise Descent

Figure 6.: Baseline TS Vertical Profiles 


\section{A. Number of Trajectory Request Successes}

Table 1 shows the comparison of the number of trajectory requests for the GenProf and Baseline TS using fourhours of aircraft traffic data in Fort Worth Center (DFW). In order to provide developers with a simple measure of trajectories generated by the two TS versions, the CTAS software was modified to calculate simple trajectory comparison statistics. For a given flight, a trajectory calculation is made during each specified time interval as the aircraft advances. Therefore, in many cases, a single problematic flight may be the cause of a number of trajectory calculation failures. For each trajectory request, the software generated a Baseline trajectory calculation and a GenProf trajectory calculation. Total trajectory requests by the client application, successful trajectories and the total number of TS trajectory calculations were tallied.

The number of client requests differs from the number of total TS calculation because the initial TS calculation may fail and the TS may handle the failure by recalculating a trajectory with modified constraints. Another statistic to note is the large difference of total TS trajectories calculations that GenProf TS has in comparison to Baseline TS. This is due to the greater number of trajectory calculation failure cases that GenProf can handle and the multiple iterations of trajectory recalculations used to handle the failure cases. Lastly, as seen in Table 3 the percentage of successful trajectories is about the same if not slightly better with the GenProf TS than the Baseline TS.

\begin{tabular}{|l|c|c|c|}
\hline $\begin{array}{l}\text { Trajectory Interface } \\
\text { Type }\end{array}$ & $\begin{array}{c}\text { Total Client Trajectory } \\
\text { Requests }\end{array}$ & $\begin{array}{c}\text { Successful Client } \\
\text { Trajectory Requests }\end{array}$ & $\begin{array}{c}\text { Total TS Trajectory } \\
\text { Calculations }\end{array}$ \\
\hline Baseline TS & 610394 & $97.40 \%$ & 611752 \\
\hline GenProf TS & 609770 & $98.44 \%$ & 743528 \\
\hline
\end{tabular}

Table 3: Baseline TS and GenProf TS Total and Successful Trajectory Requests

\section{B. Vertical Profile Comparison}

The next analysis compared the resultant trajectories generated from GenProf TS with those from the Baseline TS. This analysis identified causes of the difference between equivalent Baseline TS and GenProf TS trajectories. The trajectories from the GenProf TS and Baseline TS were compared against each other using four-hours of aircraft traffic data in Fort Worth Center (DFW) using a simple set of metrics. Based on fixed time intervals and later, fixed path distance intervals, points of the trajectories were compared. Table 4 shows the metrics and tolerances that were used for the points in the comparison.

\begin{tabular}{|l|l|}
\hline \multicolumn{1}{|c|}{ Metric } & \multicolumn{1}{c|}{ Tolerance } \\
\hline Horizontal Distance & 0.01 nautical miles \\
\hline Altitude & 10 feet \\
\hline True Air Course & 1 degree \\
\hline Ground Course & 1 degree \\
\hline True Airspeed & 2 knots \\
\hline Calibrated Airspeed & 2 knots \\
\hline Ground Speed & 2 knots \\
\hline Mach & 0.005 \\
\hline
\end{tabular}

Table 4: Metrics and Tolerances for Trajectory Comparison

A simple algorithm was used to determine the vertical profile shape for this analysis to allow for categorizing the shapes and more meaningful comparisons. This algorithm compared two consecutive altitudes while stepping through the trajectory to determine if there was an altitude change and classified them as an ascent, descent, or cruise segment. No threshold of time or distance was used to determine the type of altitude change (ascent, descent, cruise) segment. Therefore even a very small series of points with a consistent altitude would be identified as a cruise segment. The resulting altitude segment types were then sequentially chained together to produce a vertical profile shape.

The complete results of these trajectory comparisons are shown in Table 5. The total number of trajectories is shown along with the number of trajectories found that were different for each Baseline TS profile shape category. These various profile shapes are diagramed in Figure 6. A GenProf trajectory was considered different if any of the comparison metrics did not meet the specified tolerance when compared against the equivalent Baseline trajectory. 
The column with the heading of "Different Trajectories with Matching Profile Shape" is used to tally how many trajectories have matching profile shapes. A matching profile shape is one where the sequence of altitude profiles (ex. Cruise-Descent-Cruise) was the same. Differences between the two systems are not unexpected due to the process that translates Baseline TS input into GenProf input (see Figure 5).

Overall, a large number of GenProf trajectories do not match those generated from the Baseline TS. This is not surprising as the comparison tolerances were fairly tight. Also, numerous modifications to the code have been made and in many cases the differences are intended. The differences between the Baseline trajectories and GenProf trajectories do not necessarily imply that the GenProf trajectories are "wrong", but possibly just different. The differences were investigated further to determine if there were any unexpected differences, and if so, the type and amount of discrepancies. The following sections highlight a portion of the results shown in Table 5.

\begin{tabular}{|l|c|c|c|}
\hline $\begin{array}{l}\text { Baseline Vertical } \\
\text { Profile Shape }\end{array}$ & $\begin{array}{c}\text { Total } \\
\text { Trajectories }\end{array}$ & $\begin{array}{c}\text { Different } \\
\text { Trajectories }\end{array}$ & $\begin{array}{c}\text { Different Trajectories } \\
\text { With Matching Profile } \\
\text { Shape }\end{array}$ \\
\hline Overall & 32611 & 22514 & - \\
\hline Ascent Cruise & 6161 & 6161 & 16 \\
\hline Descent Cruise & 1246 & 1190 & 835 \\
\hline Cruise Descent & 1638 & 1312 & 847 \\
\hline $\begin{array}{l}\text { Cruise Descent + } \\
\text { Cruise }\end{array}$ & 6793 & 5943 & 3986 \\
\hline $\begin{array}{l}\text { Ascent Cruise } \\
\text { Descent (Cruise) }\end{array}$ & 1505 & 1505 & 745 \\
\hline $\begin{array}{l}\text { Transition Altitude } \\
\text { Descent }\end{array}$ & 284 & 272 & 161 \\
\hline $\begin{array}{l}\text { Transition Altitude } \\
\text { Ascent }\end{array}$ & 2 & 2 & 0 \\
\hline Cruise & 12271 & 3446 & 3438 \\
\hline $\begin{array}{l}\text { Transition Altitude } \\
\text { Descent + Cruise }\end{array}$ & 1316 & 1311 & 416 \\
\hline
\end{tabular}

Table 5: Comparison of Baseline TS Trajectories to GenProf TS Trajectories

\section{Baseline Ascent Cruise}

None of the GenProf trajectories matched the Baseline Ascent Cruise case (Figure 6a). Out of the 6161 trajectories, the GenProf TS models $44.4 \%$ of these as Ascent-Cruise-Descents and $44.1 \%$ as Cruise-Ascent-CruiseDescents. Profile shapes having a Cruise segment appended to ends of the two profile shapes just mentioned, made up the remaining differences that GenProf modeled for this case. The reason for these differences between Baseline and GenProf TS is because the Baseline Ascent Cruise cases were practically all departures. In the early days of CTAS, computation power was limited. Since these trajectories would descend outside the Center of focus and did not need to be analyzed outside the Center, the descent segment of these trajectories was disregarded in the Baseline TS, to conserve computation power. For these particular trajectory predictions the resultant shape for the Baseline TS was an Ascent Cruise instead of an Ascent-Cruise-Descent, which was generated by the GenProf TS.

\section{Baseline Descent Cruise}

The Cruise Descent, and the Cruise Descent + Cruise profile shape cases are all derivatives of the Baseline Descent Cruise profile shape (Figure 6b). The additional Cruise segments in the beginning of the trajectory were typically constant altitude speed changes to the assigned descent speeds. Cruise segments at the end of the trajectory may simply be where the aircraft has reached its final altitude and is cruising at a constant speed or making a final speed change.

Inspecting a small sample of "Different Trajectories With Matching Profile Shape" cases for the Baseline Cruise Descent + Cruise profile shape category, show in most instances, only altitude was the differing metric and that the maximum altitude delta was 23.1 feet. This indicated that the tolerance was too tight for the altitude comparison. Re-running the comparison test and setting the altitude tolerance to 50 feet instead of 10 feet, resulted with Baseline TS Cruise Descent + Cruise profile shape category having the number of "Different Trajectories" being reduced by 
$31.3 \%$ and the number of "Different Trajectories With Matching Profile Shape" falling by $46.2 \%$. The change in altitude tolerance generally only affected the descent cases and the overall number of comparison failures was reduced by $10.3 \%$. Further analysis is needed to determine the causes of the remaining discrepancies.

\section{Baseline Cruise}

The next case that was examined is the Baseline Cruise (Figure 6e). Virtually all the cases that had differences had the same profile shape. This is not surprising considering the simplicity of the profile. These discrepancies were found to be due to variations in the cruise speeds that were used.

\section{Enhanced Trajectory Modeling Capability}

Ensuring that the GenProf TS can generate the same trajectories as the older Baseline TS is one goal of this project. However, the main goal of this effort was to expand the types of trajectories that the TS can model beyond the limits the Baseline TS. Currently the client application cannot take full advantage of the flexibility that the GenProf TS offers. The Baseline TS to GenProf TS translation process (described in Figure 5) restricts the client to the limits of the Baseline TS expressions. A standalone TS application that reads the TS Input data directly is used to generate GenProf trajectories without any restriction. Figure 7 is an example of a Multiple Step Climb, Cruise, Step Descent trajectory that the GenProf TS is able to generate. The plot illustrates how multiple altitudes and speeds can be specified for the trajectory. This trajectory cannot be produced with the Baseline TS since it is restricted to the vertical profile shapes shown in Figure 6.

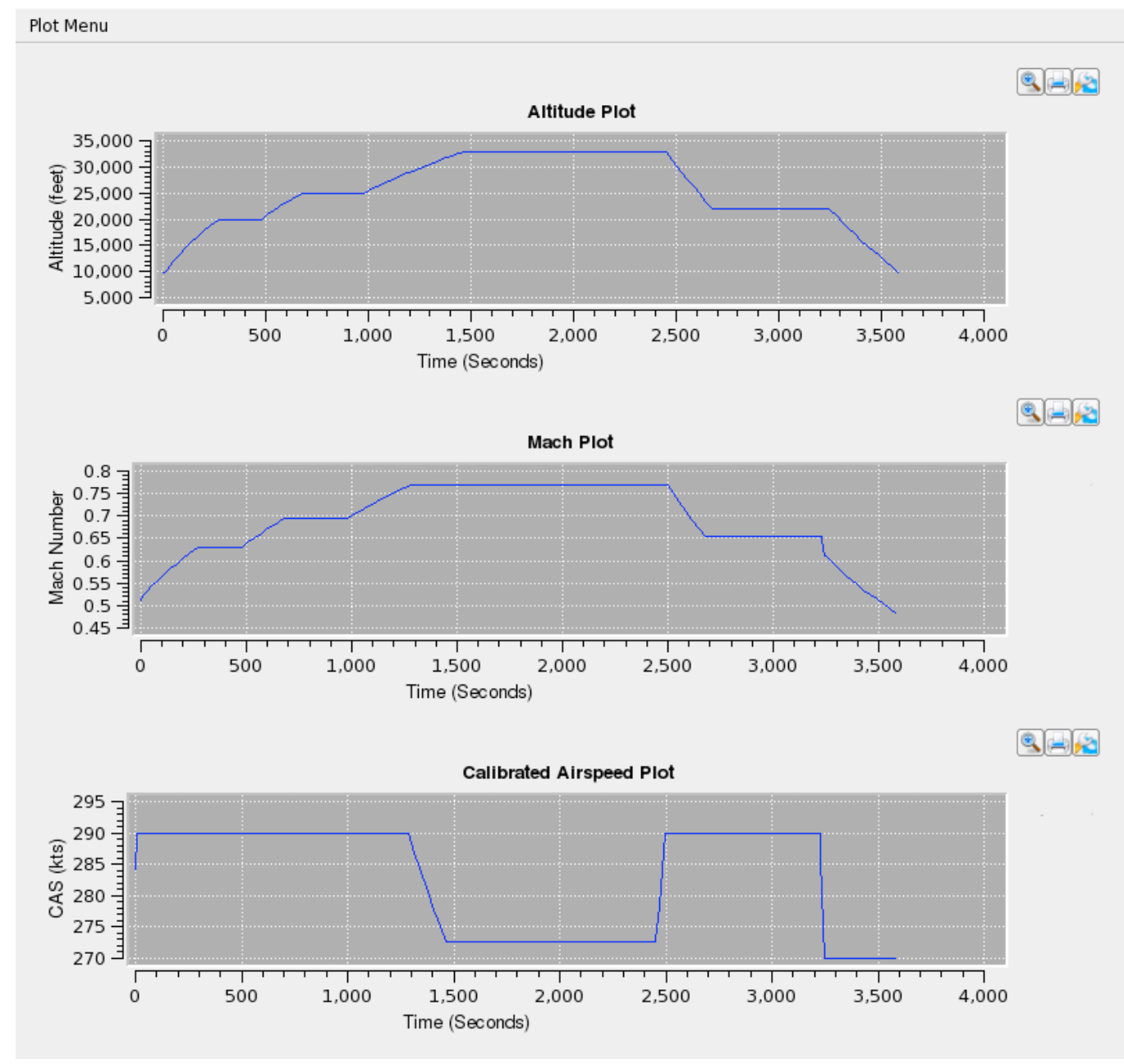

Figure 7: Multiple Step Climb, Cruise, Step Descent Trajectory Generated by GenProf 


\section{Conclusion}

A new interface to the Trajectory Synthesizer (TS) that expresses trajectory constraints in a flexible and extensible manner was developed to enhance NextGen research capability at NASA. This new GenProf interface allows an almost unlimited number of different trajectory profile types to be described, providing more realistic trajectories, and greatly enhancing its utility to NextGen researchers. Furthermore, the interoperability aspect of GenProf may increase the scope of its usage outside that of the TS.

Architectural changes to the software moved much of the decision control logic out of the legacy Baseline TS software to client applications, allowing the client process to be able to interpret the trajectory results and make adjustments to the trajectory request and resubmit the request, if necessary. The trajectory computation was isolated to the TS. Other new capabilities added to the TS improved its maintainability and provides the flexibility needed to meet the requirements of various client tools (such as trajectory accuracy and computation performance) that utilize the TS.

Trajectory predictions from the Baseline TS and GenProf versions were compared and the results, in terms of the number of successful trajectory requests as well as a comparison of the output trajectories, was analyzed. The test that compared the number of successful trajectory requests showed that GenProf TS was able to produce approximately the same percentage of successful trajectories as Baseline TS. Comparing equivalent output trajectories generated by the Baseline TS against those of the GenProf TS showed that there were a large number of differences. A number of differences appear to be related to differences in assigned speeds. Other speed related issues such as the point at which the speeds are captured are another source of difference. These can either be different points in the flight segment (i.e. beginning or end of a segment) or whether the speed is being captured during an altitude change or not. Altitude related differences were evident when examining the Descent Cruise profiles cases. Factors involved with these differences were the altitude tolerances used in comparing the trajectories. Lastly, in the case of the Climb-Cruise-Descent trajectories, the difference was because the Baseline TS ignored the descent portion of the trajectory. These results will be examined further in future analysis.

\section{Acknowledgements}

The authors would like to thank Karen Cate from NASA Ames Research Center for providing technical guidance and historical insight.

\section{References}

\footnotetext{
${ }^{1}$ Joint Planning and Development Office, "Next Generation Air Transportation System Integrated Plan,” Dec. $12,2004$.

${ }^{2}$ National Aeronautics and Space Administration, “Airspace Systems Program NextGen-Airspace Project Fy2009 Project Plan" Dec., 2008

3 National Aeronautics and Space Administration, "Next Generation Air Transportation System (NGATS) Air Traffic Management (ATM)-Airspace Project Reference Material” Jun., 2006

${ }^{4}$ Erzberger, H., and Lee, H. Q., "Constrained Optimum Trajectories with Specified Range," Journal of Guidance and Control, Vol. 3, No. 1, 1980, pp. 78-85.

${ }^{5}$ Slattery, R., and Zhao, Y., "Trajectory Synthesis for Air Traffic Automation," Journal of Guidance, Control and Dynamics, Vol. 20, No. 2, March/April 1997, pp. 232-238

${ }^{6}$ Nedell, W., Erzberger, H., and Neuman, F., "The Traffic Management Advisor," 1990 American Control Conference, San Diego, CA, May 1990.

${ }^{7}$ Hoang, T., and Swenson, H., "The Challenges of Field Testing of the Traffic Management Advisor in an Operational Air Traffic Control Facility," AIAA Guidance, Navigation and Control Conference, New Orleans, LA, Aug. 1997.

${ }^{8}$ Erzberger, H., "Transforming the NAS: The Next Generation Air Traffic Control System," 24th International Congress of the Aeronautical Sciences (ICAS 2004), Yokohama, Japan, August 29 - September 3, 2004.

9 McNally, D., and Gong, C., "Concept and Laboratory Analysis of Trajectory-Based Automation for Separation Assurance," AIAA-2006-6600, AIAA Guidance, Navigation and Control Conference and Exhibit, Keystone, CO, 21-24 Aug. 2006.

${ }^{10}$ Gouldner, D., Boerner, S., "Evaluating NGATS Research Priorities at JPDO," Proceedings of the 6th AIAA ATIO Conference, Wichita, KS, Sept. 2006.

${ }^{11}$ Coppenbarger, R. A., Mead, R. W., and Sweet, D. N., "Field Evaluation of the Tailored Arrivals Concept for Datalink-Enabled Continuous Descent Approach," AIAA-2007-7778, AIAA Aviation Technology, Integration and Operations Conference (ATIO), Belfast, Northern Ireland, 18-20 Sep. 2007.
} 
${ }^{12}$ Green, S. M., Davis, T. J., and Erzberger, H., "A Piloted Simulator Evaluation of a Ground-Based 4D Descent Advisor Algorithm," Proceedings of the AIAA Conference on Guidance, Navigation, and Control, Monterey, CA, Aug. 1987, pp. 11731180 .

${ }^{13}$ Tobias, L., Volckers, U., and Erzberger, H., "Controller Evaluations of the Descent Advisor Automation Aid," NASA TM$102197,1989$.

${ }^{14}$ Erzberger, H., Davis, T. J., and Green, S. M., "Design of Center-TRACON Automation System," AGARD Meeting on Machine Intelligence in Air Traffic Management, Berlin, Germany, 11-14 May 1993.

${ }^{15}$ Swenson, H. N., Hoang, T., Engelland, S., Vincent, D., Sanders, T., Sanford, B., and Heere, K., "Design and Operational Evaluation of the Traffic Management Advisor at the Fort Worth Air Route Traffic Control Center," 1st USA/Europe Air Traffic Management R\&D Seminar, Saclay, France, June 1997.

${ }^{16}$ Green, S. M., Vivona, R., and Sanford, B., "Descent Advisor Preliminary Field Test," AIAA-95-3368, AIAA Guidance, Navigation, and Control Conference, Aug. 1995.

${ }^{17}$ Mueller, K. T., and Robinson III, J. E., "Final Approach Spacing Tool (FAST) Velocity Accuracy Performance Analysis," AIAA Guidance, Navigation, and Control Conference, Boston, MA, Aug. 1998.

${ }^{18}$ McNally, B. D., Bach, R. E., and Chan, W., "Field Test Evaluation of the CTAS Conflict Prediction and Trial Planning Capability," AIAA Guidance, Navigation, and Control Conference, Boston, MA, 10-12 Aug. 1998.

${ }^{19}$ Federal Aviation Administration: Office of System Capacity, 1998 Aviation Capacity Enhancement Plan, December 1998.

${ }^{20}$ Oseguera, R. M., and Williams, D. H., "Flight Evaluation of the CTAS Descent Advisor Trajectory Prediction," American Control Conference, Seattle, WA, 21-23 June 1995.

${ }^{21}$ Vivona, R.A., Cate, K.T., and Green, S.M.: "Abstraction Techniques for Capturing and Comparing Trajectory Predictor Capabilities and Requirements", AIAA GN\&C Conference, Honolulu, HI, 2008

${ }^{22}$ Coppenbarger, R.A., Mead, R.W., and Sweet, D.N., "Field Evaluation of the Tailored Arrivals Concept for

Datalink-Enabled Continuous Descent Approach," 7th AIAA Aviation Technology, Integration and

Operations Conference, Belfast, Northern Ireland, Sept. 2007.

${ }_{23}$ Jardin, M. R., and Erzberger, H., "Atmospheric Data Acquisition and Interpolation for Enhanced Trajectory-Prediction Accuracy in the Center-TRACON Automation System," AIAA-96-0271, 34th Aerospace Sciences Meeting \& Exhibit, Reno, NV, 15-18 Jan. 1996. 